\title{
Erziehung zur Moralität in einer komplexen Welt
}

\author{
Thomas Rucker
}

Eingegangen: 15. Februar 2021 / Überarbeitet: 6. April 2021 / Angenommen: 27. Mai 2021 / Online publiziert: 1. September 2021

(C) Der/die Autor(en) 2021

Zusammenfassung In diesem Beitrag wird das Problem behandelt, wie eine Erziehung zur Moralität in einer komplexen Welt sinnvoll konzipiert werden kann. Um eine Antwort auf diese Frage zu finden, wird zunächst ein von Michael Hand entwickelter Lösungsvorschlag für die Problemstellung rekonstruiert. Daraufhin wird der Vorschlag auf den Prüfstand gestellt und gezeigt, dass dieser ein in der Pädagogik bereits erreichtes Problemniveau in spezifischer Hinsicht unterschreitet. Im Mittelpunkt stehen zwei Einwände, die als das Kombinationsdefizit erzieherischer Grundformen und als der Vorwurf aus der Imperfektheit der Ethik bezeichnet werden. Schließlich wird argumentiert, dass die Theorie nichtaffirmativer Moralerziehung die Möglichkeit eröffnet, die Problemkonstellation zu umgehen, in die Hands Theorieentwurf hineinführt. Umgekehrt wird die Position vertreten, dass dieser Entwurf Anlass dazu gibt, die Theorie nichtaffirmativer Moralerziehung selbst nicht unbefragt zu affirmieren, sondern an deren Weiterentwicklung zu arbeiten, um moralische Erziehung in einer komplexen Welt zukünftig differenzierter zu konzipieren.

Schlüsselwörter Erziehung · Moralität · Komplexität · Imperfektheit · Achtung

PD Dr. Thomas Rucker $(\bowtie)$

Institut für Erziehungswissenschaft, Abteilung für Allgemeine und Historische

Erziehungswissenschaft, Universität Bern, Fabrikstr. 8, 3012 Bern, Schweiz

E-Mail: thomas.rucker@edu.unibe.ch 


\title{
Education for morality in a complex world
}

\begin{abstract}
In this paper I shall address the problem of how education for morality can be reasonably conceived in a complex world. To find an answer to this question, I will first reconstruct a proposed solution to the problem developed by Michael Hand. I will then subject the proposal to scrutiny and show that it undermines an already achieved educational-theoretical level. The focus is on two objections, which I call the combination deficit of basic educational forms on the one hand and the objection from the imperfection of ethics on the other hand. Finally, I argue that the theory of non-affirmative moral education opens up the possibility of circumventing the problems that Hand's theory entails. Conversely, I argue that Hand's theory gives reason not to uncritically affirm the theory of non-affirmative moral education, but to work on its further development in order to conceptualize moral education in a complex world in a more differentiated way.
\end{abstract}

Keywords Education $\cdot$ Morality $\cdot$ Complexity $\cdot$ Imperfection $\cdot$ Recognition

\section{Das Problem}

Die Krise der liberalen Demokratie, der Klimawandel, die Entstehung und Stabilisierung einer Kultur der Digitalität, nicht zuletzt die Frage nach einem sinnvollen Umgang mit der Coronapandemie - jeweils handelt es sich um Ereignisse, an denen moralisch relevante Aspekte ausgemacht werden können. Entsprechend werden Menschen zu einem moralisch gerechtfertigten Handeln aufgerufen und etwa da$\mathrm{zu}$ aufgefordert, Masken zu tragen, um Andere vor einer potenziellen Infektion zu schützen, oder einen nachhaltigen Lebensstil zu pflegen, um ,ihren“ Beitrag dazu zu leisten, eine drohenden Klimakatastrophe abzuwenden. Dabei wird nolens volens vorausgesetzt, dass Menschen dazu bereit und fähig sind, moralische Ansprüche als solche zu erkennen sowie den jeweiligen Einsichten im Handeln zu entsprechen. Diese Annahme ist - pädagogisch betrachtet - keine Selbstverständlichkeit. Aus pädagogischer Warte kommt Moralität vielmehr als etwas in den Blick, das nicht einfach gegeben, sondern in Entwicklung begriffen und hierbei auf Erziehung angewiesen ist.

Bei der Frage, wie eine Erziehung zur Moralität sinnvoll konzipiert werden kann, handelt es sich um eine Problemvorgabe der Tradition (vgl. Herbart 1964a [1804], S. 259), der bis dato der Status eines Dauerproblems pädagogischer Theoriebildung zukommt (vgl. Thimm 2007). Einer Erziehungswissenschaft, die an der Idee eines theoretischen Fortschritts festhält, kommt in diesem Zusammenhang u. a. die Aufgabe zu, Beschreibungen einer Erziehung zur Moralität - auch immer wieder neu auf den Prüfstand zu stellen. Dieser Aufgabenstellung liegt die Annahme zugrunde, dass Fortschritt in theoretischen Fragen möglich ist - und zwar dadurch, dass Alternativen aus einer Problematisierung überkommener Entwürfe heraus entwickelt und diese Beschreibungen selbst wiederum der Konfrontation mit alternativen Entwürfen ausgesetzt werden. 
Diesem Anspruch an wissenschaftliche Theoriebildung möchte ich im Folgenden dadurch Rechnung tragen, dass ich eine spezifische Problemstellung in den Fokus der Aufmerksamkeit rücke. Diese Problemstellung ergibt sich dann, wenn wir den traditionellen Topos einer Erziehung zur Moralität mit einer aktuellen Selbstbeschreibung moderner Gesellschaften als komplexen Gesellschaften in Beziehung setzen - einer Selbstbeschreibung, die eine zeitgemäße Theorie der moralischen Erziehung vor spezifische Herausforderungen stellt. Das Problem lautet, wie eine Erziehung zur Moralität sinnvoll konzipiert werden kann, wenn wir davon ausgehen, dass das Aufwachsen in modernen Gesellschaften unter den Bedingungen von Komplexität seinen Ort hat.

Diese Problemstellung impliziert eine Reihe von begrifflichen Festlegungen, die es zunächst zu erläutern gilt: Unter Moral verstehe ich Regeln, die mit dem Anspruch verknüpft werden, dass alle Menschen sich auf sie verpflichten sollen. Zugespitzt formuliert: „Eine moralische Position vertritt nur derjenige, der von den anderen fordert dasselbe zu finden" (Tugendhat 1993, S. 64). Moral wird damit in Abhängigkeit von der Einstellung bestimmt, die eine Person in Bezug auf eine bestimmte Regel einnimmt. Eine Regel ist folglich nicht , an sich“ eine moralische oder eine nichtmoralische Regel. Vielmehr kann ein und dieselbe Regel (z. B. ,Du sollst andere Menschen nicht betrügen!', ,Du sollst keine Tiere misshandeln!' oder ,Du sollst dich vegetarisch ernähren!') für eine Person als eine moralische und für eine andere Person als eine nichtmoralische Regel fungieren. Eine Regel avanciert zu einer moralischen Regel genau dann, wenn diese eine Sollensforderung zum Ausdruck bringt, die an alle Menschen adressiert ist (was freilich auch diejenige Person mit einschließt, die die jeweilige Sollensforderung erhebt). Moralische Regeln geben eine Antwort auf die Frage, was wir uns wechselseitig als Menschen schulden (vgl. Scanlon 1998), und erheben in diesem Sinne einen kategorischen Geltungsanspruch. Der Vorschlag, dass moralische Regeln mit einem Anspruch auf unbedingte Verbindlichkeit verknüpft sind, besagt nicht, dass ein solcher Geltungsanspruch immer auch bewährt werden kann. Der hier verwendete Begriff von Moral lässt es vielmehr zu, auch von ungerechtfertigten moralischen Regeln zu sprechen, womit die Frage aufgeworfen ist, anhand welcher Kriterien der Rechtfertigungsstatus einer moralischen Regel beurteilt werden könnte, sollte oder gar müsste.

Der vorgeschlagene Begriff von Moral hat zwei Vorzüge: Zum einen erlaubt es dieser Begriff, eine Grenze zwischen moralischen und nichtmoralischen Regeln zu ziehen - z. B. sittlichen Regeln, die für eine bestimmte Gruppe Geltung beanspruchen, oder Ansprüchen, die für die individuelle Lebensführung als maßgeblich in Anschlag gebracht werden, und die darüber Auskunft geben, wie man selbst leben will, um ein gelingendes Leben zu führen. Dies wiederum eröffnet die Möglichkeit, verschiedene Bereiche des Normativen zu unterscheiden und - damit verbunden - den Widerstreit zwischen verschiedenen Formen der Regelorientierung in den Blick zu nehmen, wie er für moderne Gesellschaften typisch zu sein scheint (vgl. Birnbacher 2013, S. 53 f.). Ein besonderes Potenzial der hier vorgenommenen Begriffsbestimmung scheint mir hierbei darin zu bestehen, dass die Frage nach den Grenzen von moralischen Ansprüchen selbst noch als ein Streitthema erfasst werden kann. Zum anderen erlaubt es der vorgeschlagene Moralbegriff, von verschiedenen Moralen zu sprechen, indem moralische Regeln nicht bereits vorab z.B. als 
universalisierbare Ansprüche begriffen werden, wie dies in kantscher Tradition der Fall ist. Verallgemeinerbarkeit wäre vielmehr als ein Kriterium zu begreifen, anhand dessen in dieser Tradition gerechtfertigte von ungerechtfertigten moralischen Regeln unterschieden werden, nicht aber als ein Kriterium, um moralische Ansprüche von anderen Formen der Regelorientierung zu differenzieren. In diesem Sinne beansprucht der vorgeschlagene Moralbegriff Neutralität gegenüber verschiedenen Moralvorstellungen, und dabei insbesondere auch gegenüber unterschiedlichen moralphilosophischen Traditionen (vgl. Koller 1983, S. 270). Dies wiederum eröffnet die Möglichkeit, den Widerstreit zwischen verschiedenen moralischen Positionen, wie wir ihn in modernen Gesellschaften ebenfalls beobachten können, zu thematisieren, statt diesen definitorisch (semantisch) vorzuentscheiden.

Die Möglichkeit der Thematisierung beider Formen von Widerstreit ist gerade dann von Bedeutung, wenn man an einer Klärung der Frage interessiert ist, wie eine Erziehung zur Moralität in einer komplexen Welt sinnvoll beschrieben werden kann. Mit dem Begriff der Komplexität werden nämlich Problemstellungen bezeichnet, die in der Öffentlichkeit kontrovers diskutiert werden, ohne dass wir um eine Regel wissen, die es erlauben würde, eine Position für allgemeinverbindlich zu erklären (vgl. Nassehi 2017). Auf Problemstellungen dieser Art stoßen wir nicht nur, aber auch dann, wenn wir uns der Frage zuwenden, was wir uns wechselseitig als Menschen schulden. Auch in Sachen Moral haben wir es mit Dissensen zwischen verschiedenen Positionen zu tun, für die jeweils gute Gründe angeführt werden können, ohne das ein bester Grund zur Verfügung steht, der den Dissens auflösen könnte (vgl. Luhmann 1998, S. 184).

Es ist diese Komplexität der Moral, wie man mit Charles Larmore (1994) formulieren könnte, der ich im Folgenden meine Aufmerksamkeit widmen möchte. Aus pädagogischer Warte stellt sich hier nämlich die Frage, ob und, falls ja, wie eine Erziehung zur Moralität unter diesen Bedingungen sinnvoll konzipiert werden kann. Um eine Antwort auf diese Frage zu finden, werde ich zunächst einen von Michael Hand $(2014,2018)$ entwickelten Lösungsvorschlag für die besagte Problemstellung rekonstruieren, der international aktuell intensiv diskutiert wird (vgl. u.a. Clayton und Stevens 2019; Kotzee 2020; White 2017). Dies ist nicht weiter verwunderlich, zeigt Hands Theorieentwurf doch ein Problemniveau an, das nicht unterschritten werden sollte, wenn das Problem einer Erziehung zur Moralität in einer komplexen Welt heute formuliert und bearbeitet wird. In diesem Beitrag möchte ich den Versuch unternehmen, nicht bei diesem Problemniveau stehen zu bleiben, sondern an dessen Weiterentwicklung zu arbeiten. ${ }^{1}$

\footnotetext{
${ }^{1}$ Eine solche Arbeit kann in dem hier zur Verfügung stehenden Rahmen freilich nur unter starken Restriktionen erfolgen. So diskutiere ich in diesem Beitrag ausschließlich Theorieentwürfe, in denen eine Erziehung zur Moralität explizit in Bezug auf das Problem der Komplexität thematisiert wird. In diesem Sinne handelt es sich bei der Auswahl der theoretischen Positionen um eine durch Plausibilitätsargumente gestützte Auswahl, zu der freilich Alternativen denkbar wären. Zugleich ist darin ein Grund dafür zu sehen, weshalb die in der Erziehungswissenschaft bis heute geführte Debatte im Anschluss an die Theorie der Entwicklung des moralischen Urteils von Lawrence Kohlberg im Folgenden ausgeklammert bleibt.
} 


\section{Ein Vorschlag}

Eine Erziehung zur Moralität wird gemeinhin als etwas angesehen, dass notwendig ist, damit Heranwachsende sich zu Menschen entwickeln, die sich auf moralische Regeln verpflichten und diese Regeln darüber hinaus für gerechtfertigt halten. Zugleich kann in modernen Gesellschaften ein ,reasonable disagreement about the content and justification of morality“ (Hand 2018, S. 1) ausgemacht werden. Menschen sind sich aus guten Gründen uneins darüber, was sie sich wechselseitig als Menschen schulden. Darüber hinaus besteht ein vernünftiger Pluralismus an Positionen hinsichtlich der Frage, warum bestimmte Ansprüche als moralisch geboten, verboten oder neutral einzustufen sind.

Diese Konstellation bringt eine Erziehung zur Moralität in eine prekäre Lage. Ist nämlich in Sachen Moral ein vernünftiger Dissens feststellbar, dann, so Hands These, müsse jeder Versuch, die Heranwachsenden auf bestimmte Positionen festzulegen, als Indoktrination gewertet werden. „It looks as though any attempt to persuade children of the authority of a selected moral code, when there are perfectly respectable alternatives available, is bound to be indoctrinatory" (Hand 2018, S. 1). Wenn es nämlich richtig ist, dass Menschen sich in Bezug auf den Inhalt und die Rechtfertigung moralischer Ansprüche aus guten Gründen uneins sind, so bedeutet dies zugleich, dass pädagogische Akteure an einem bestimmten Punkt nicht mehr daran vorbei kommen, den Dialog - im Sinne der argumentativen Rede - mit den Heranwachsenden aufzugeben und $\mathrm{zu}$ anderen, eben nichtargumentativen Methoden und Mitteln zu greifen, um diese dazu zu veranlassen, bestimmte moralische Ansprüche nicht bloß zu befolgen, sondern diese zugleich für gerechtfertigt zu halten (Überredung mittels Sympathie, Verschweigen von Alternativen, Verbreitung von Falschinformationen etc.). Dies aber bedeute, so Hand, nichts anderes als eine Indoktrination der Heranwachsenden, denn ,to indoctrinate someone is to impart beliefs to her in such a way that she comes to hold them non-rationally, on some other basis than the force of relevant evidence and argument" (Hand 2018, S. 6).

Ein Ausweg aus dieser Situation kommt dann in den Blick, wenn man einen bestimmten Irrtum aufdeckt. Dieser Irrtum besteht Hand zufolge darin, dass es schlichtweg ein Fehler sei, wollte man aus dem Umstand, dass in der Moral vieles umstritten ist, darauf schließen, dass wir es in der Moral nur mit Widerstreit zu tun hätten. Einer solchen Beschreibung stellt Hand zwei Thesen entgegen: Die erste These lautet, dass es einen ,Kernbestand ' an Regeln gebe, deren Status als moralische Regeln niemand ernsthaft in Frage stellt. Zu einer solchen ,Minimalmoral' zählen sowohl eine Reihe von Verboten, nämlich Menschen nicht zu ermorden, zu bestehlen, zu erpressen, zu belügen und zu betrügen, als auch eine Reihe von Geboten, nämlich Menschen fair zu behandeln, die eigenen Versprechen zu halten sowie Not leidenden Menschen zu helfen. Hand geht nun aber noch einen Schritt weiter. Die zweite These besagt nämlich, dass es auch ein Argument gebe, das diesen Kernbestand an moralischen Regeln gleichsam ,hieb- und stichfest' rechtfertigt (vgl. Hand 2018, S. $59 \mathrm{ff}$.).

Ich hatte moralische Regeln eingangs als solche Regeln bestimmt, die mit einem allgemeinen Verbindlichkeitsanspruch verknüpft sind. Hieraus folgt, dass es für eine Rechtfertigung moralischer Regeln nicht ausreicht, zu zeigen, dass sich einzelne 
Personen auf die jeweiligen Regeln verpflichten sollen. Vielmehr bedarf es eines Arguments, das zeigt, dass jeder Mensch sich auf die besagten Regeln verpflichten sollte. Das Argument, das Hand anbietet, ist keineswegs neu, sondern in der Ethik unter dem Namen ,Kontraktualismus “ wohl bekannt. Hand stellt sich ausdrücklich in eine Tradition für die u. a. Philosophen wie Thomas Hobbes, David Hume, H.L.A. Hart oder J.L. Mackie stehen. ${ }^{2}$ Der Kern des Arguments lautet, dass moralische Regeln auf ein Dauerproblem menschlicher Existenz und Koexistenz reagieren, das Hand im Anschluss an David Copp (2009) als das Problem der Sozialität bezeichnet.

Das Problem der Sozialität entsteht, weil die menschliche Lebensform durch drei Merkmale gekennzeichnet ist, die in ihrem Zusammenhang die permanente Gefahr eines Ausbruchs mehr oder minder großer Konflikte mit sich bringen: Menschen begegnen sich prinzipiell als verletzliche Wesen. Darüber hinaus verfügen sie gegenüber Fremden nur über ein begrenztes Maß an Sympathie. Wir sind dazu geneigt, das eigene Wohlergehen sowie das Wohlergehen derjenigen Personen, die uns nahestehen, dem Wohlergehen Fremder überzuordnen - was nicht bedeutet, dass Menschen prinzipiell egoistisch eingestellt seien. Die Ressourcen, die Menschen zur Verfügung haben, sind schließlich nicht ausreichend, um die Interessen von allen zu befriedigen. Menschen sind deshalb gleichsam zu einem Wettbewerb mit anderen Menschen um den Zugang zu knappen Gütern gezwungen.

Um den Ausbruch von Konflikten, die das Zusammenleben (und vielleicht sogar das Überleben) der Menschen bedrohen, einzudämmen, ist Hand zufolge eine Verpflichtung auf elementare moralische Regeln erforderlich. Diese verhindern z. B., dass Menschen sich aufgrund von nur begrenzter Sympathie wechselseitig verletzen, oder dass im Wettbewerb um knappe Ressourcen Verlässlichkeit gewahrt bleibt. In diesem Sinne beruht die Begründungsfigur auf der Annahme, dass der soziale Zweck moralischer Regeln diese Regeln zugleich rechtfertigt, eben weil jeder ein Interesse daran hat, dass das Zusammenleben möglichst konfliktfrei funktioniert. Aufgrund dieses Interesses - so die Pointe - haben alle einen guten Grund, sich auf bestimmte moralische Regeln zu verpflichten.

Moralität besitzt zwei Aspekte, nämlich ,subscribing to moral standards and believing them to be justified" (Hand 2018, S. 15; Hv.i. O.). Analog hierzu unterscheidet Hand zwischen zwei Grundformen von moralischer Erziehung. Die erste Form bezeichnet er als moral formation. Die Heranwachsenden sollen darin unterstützt werden, die subjektiven Voraussetzungen zu entwickeln, um bestimmten moralischen Regeln im Handeln zu entsprechen. Hand unterscheidet fünf Methoden einer solche Haltungs- bzw. Charaktererziehung, nämlich das Vorschreiben von Regeln, die Belohnung für das Beachten von Regeln, die Bestrafung für die Missachtung von Regeln, das Vorleben der Beachtung von Regeln sowie das Vorleben angemessener Reaktionen in Bezug auf die Beachtung oder Missachtung von Regeln durch Andere (vgl. Hand 2018, S. $30 \mathrm{ff}$.).

Die zweite Form von moralischer Erziehung bezeichnet Hand als moral inquiry (vgl. Hand 2018, S. $37 \mathrm{ff}$.). Hier geht es nicht darum, Heranwachsende in ihrem

\footnotetext{
2 Die in der Tradition des Kontraktualismus bedeutsame Differenz von Moral und Recht bleibt in Hands Theorieentwurf unberücksichtigt - ein Befund, der auch mit Blick auf die Pädagogik im deutschsprachigen Raum nicht untypisch zu sein scheint (vgl. Tenorth 2015, S. 13f.).
} 
Handeln zu unterstützen. Vielmehr ist moralische Erziehung in diesem Sinne darauf gerichtet, moralische Regeln und ihren Rechtfertigungsstatus zu thematisieren. In diesem Zusammenhang unterscheidet Hand noch einmal zwischen einer directive moral inquiry und einer non-directive moral inquiry, wobei die Überzeugungsabsicht als Unterscheidungskriterium fungiert. Im Falle einer direktiven Moralerziehung ist die Thematisierung des Rechtfertigungsstatus von der Absicht geleitet, dass die Heranwachsenden eine bestimmte Regel als gerechtfertigt oder als nicht gerechtfertigt akzeptieren sollen. Nichtdirektiv ist eine Moralerziehung hingegen dann, wenn keine entsprechende Absicht im Spiel ist. Hier werden moralische Regeln und ihre Begründbarkeit zwar ebenfalls thematisiert, es wird jedoch offen gelassen, welche Regeln und welche Begründungen die Heranwachsenden für sich selbst als maßgeblich bestimmen.

Es dürfte klar sein, dass es die direktive Moralerziehung ist, die Gefahr läuft, in Indoktrination abzugleiten - nämlich dann, wenn moralische Regeln als gerechtfertigt bzw. ungerechtfertigt behandelt werden, obgleich es vernünftige Alternativen gibt. Es mag zwar auch im Rahmen der nichtdirektiven Moralerziehung dazu kommen, dass sich Heranwachsende Positionen auf nichtrationale Art und Weise aneignen. Indem die Frage der Rechtfertigung moralischer Regeln als eine offene Frage thematisiert wird, ist nichtdirektive Moralerziehung jedoch von vorneherein darauf gerichtet, das Risiko für Indoktrination zu minimieren. Hand spricht darüber hinaus die Haltungs- bzw. Charaktererziehung von jeglichem Indoktrinationsverdacht frei: „The process of cultivating moral attidutes and dispositions in children cannot itself be indoctrination because attitudes and dispositions are not beliefs, and therefore not the sorts of things that can be indoctrinated" (Hand 2018, S. $40 \mathrm{f}$.).

Es stellt sich nun die Frage, wie eine Erziehung zur Moralität in einer komplexen Welt aus der Warte von Hands Theorie beschrieben werden kann. Um eine sinnvolle Antwort auf diese Frage zu geben, ist es erforderlich, den spezifischen Rechtfertigungsstatus moralischer Regeln zu berücksichtigen. Entsprechend unterscheidet Hand zwischen gerechtfertigten moralischen Regeln, moralischen Regeln, deren Rechtfertigungsstatus umstritten ist, und moralischen Regeln, für die es keine Rechtfertigung gibt, d.h. die unbegründet sind. Diesen drei Arten von Regeln ordnet Hand verschiedene Grundformen moralischer Erziehung zu. In Bezug auf Regeln, die für ein konfliktfreies Zusammenleben der Menschen als unverzichtbar gelten können, votiert Hand für eine Kombination von Haltungserziehung und direktiver Moralerziehung. Der Anspruch lautet, dass Heranwachsende sich im Handeln auf elementare moralische Regeln verpflichten und diese Regel darüber hinaus für gerechtfertigt halten sollen. Demgegenüber spricht sich Hand im Falle von Regeln, über deren Rechtfertigung eine vernünftige Meinungsverschiedenheit besteht, für eine nichtdirektive Moralerziehung aus. Anspruch ist es, Heranwachsende bei der Entwicklung eigener Urteile in der Auseinandersetzung mit verschiedenen vernünftigen Optionen zu unterstützen. Hingegen vertritt Hand die Position, das ungerechtfertigte moralische Regeln in der Erziehung nicht kontrovers, d.h. mit offenem Ausgang diskutiert werden sollten (für ein Beispiel vgl. Hand 2007). Hand empfiehlt vielmehr ein Handeln, das darauf gerichtet ist, Heranwachsende davon zu überzeugen, dass für die jeweiligen Regeln keine guten Gründe angeführt werden können. Auf 
ungerechtfertigte moralische Regeln sollte folglich mit direktiver Moralerziehung reagiert werden (vgl. Hand 2018, S. 76ff.).

Die von Hand entwickelte Systematik wird von diesem dadurch begründet, dass er mögliche Alternativen als problematisch auszuweisen sucht. So wird etwa der Vorschlag, moralische Erziehung allein als Haltungserziehung zu beschreiben, mit dem Argument zurückgewiesen, dass das Wissen um gerechtfertigte moralische Regeln dazu beitragen würde, unsere Verpflichtung auf moralische Standards zu stabilisieren. Aus diesem Grund sei moral formation auf die Ergänzung um eine direktive Moralerziehung angewiesen (vgl. Hand 2018, S. 44). Es wäre allerdings ebenso problematisch, moralische Erziehung auf eine direktive Moralerziehung zu reduzieren. Das Argument lautet in diesem Fall, dass die Thematisierung moralischer Regeln und ihres Rechtfertigungsstatus als solche nicht dazu beiträgt, dass Heranwachsende lernen, den jeweiligen Regeln auch im Handeln zu entsprechen. Eben dies sei aber der Sinn einer Erziehung zur Moralität (vgl. Hand 2018, S. 5 f.). Diese kann aber auch nicht in einer Kombination von Haltungserziehung und direktiver Moralerziehung aufgehen - und zwar deshalb nicht, weil im Bereich der Moral eben auch vernünftiger Dissens beobachtet werden kann. Hier kann moralische Erziehung nicht direktiv verfahren, ohne in Indoktrination abzugleiten. Stattdessen bedarf es einer nichtdirektiven Moralerziehung, die darauf gerichtet ist, verschiedene moralische Positionen kontrovers, d.h. mit offenem Ausgang zu thematisieren, und dem Einzelnen dabei zu helfen, eine eigene Position zu finden. Schließlich könne eine Erziehung zur Moralität aber auch nicht durchgängig als nichtdirektive Moralerziehung konzipiert werden. Der Grund lautet, dass eine solche Erziehung Gefahr liefe, Heranwachsende in Richtung eines moralischen Agnostizismus zu ,stupsen“ (vgl. Hand 2018, S. 12 u. S. 82). Hand greift hier auf eine Annahme zurück, deren Prüfung letztlich nach einer empirischen Untersuchung verlangt, nämlich dass eine permanente offene Thematisierung moralischer Regeln und ihres Rechtfertigungsstatus die Wahrscheinlichkeit dafür erhöht, dass Heranwachsende die Überzeugung entwickeln, sich in moralischer Hinsicht nicht festlegen zu können und zu wollen. Sollte dies zutreffen, so würde eine Moralerziehung, die rein nichtdirektiv ausgerichtet ist, den eigentlichen Anspruch moralischer Erziehung unterlaufen, nämlich dass Heranwachsende sich auf überindividuelle Verbindlichkeiten verpflichten sollen. Diese Gefahr könne nur gebannt werden, wenn nichtdirektive Moralerziehung um eine direktive Moralerziehung ergänzt wird, so dass Heranwachsende erkennen können, dass es durchaus definitive Antworten auf die Frage gibt, was wir uns wechselseitig als Menschen schulden (vgl. Hand 2018, S. 82).

\section{Zwei Einwände}

Hands Theorieentwurf ist in den letzten Jahren intensiv diskutiert worden. In diesem Zusammenhang sind eine Reihe von Einwänden erhoben worden, etwa in Bezug auf die fehlende Berücksichtigung empirischer Forschungsbefunde (de Ruyter 2019, S. $644 \mathrm{ff}$.), in Bezug auf einen vermeintlich allzu restriktiven Begriff der Indoktrination (Copp 2016, S. 151), oder auch in Bezug auf eine bislang fehlende Klärung der Überführung der ,Problem-der-Sozialität-Argumentation“ in Formen, die von 
Heranwachsenden unterschiedlicher Lebensalter verstanden werden können (White 2017, S. 340). Ich möchte im Folgenden nicht näher auf diese und andere Einwände eingehen oder mich gar an einer Kritik dieser Kritiken versuchen. Statt einer Reproduktion bereits bekannter Einwände geht es mir darum, zwei eigene Einwände zu entwickeln, die zeigen, dass Hands Theorie in spezifischer Hinsicht hinter ein in der Pädagogik bereits erreichtes Problemniveau zurückfällt.

Der erste Einwand bezieht sich auf ein Kombinationsdefizit von erzieherischen Grundformen, das in Hands Entwurf ausgemacht werden kann. Wie erwähnt, gebraucht Hand den Indoktrinationsbegriff ausdrücklich nicht dazu, um problematische Ausprägungen einer moral formation zu bezeichnen. Im Gegenteil: Hand spricht die Haltungs- bzw. Charaktererziehung von jedem Indoktrinationsverdacht frei. Allerdings führt Hand auch keine anderen Begriffe ein, um defizitäre Formen von moral formation in den Blick zu rücken. Diese erste Grundform von moralischer Erziehung scheint für ihn weit weniger problematisch als die einer (directive) moral inquiry zu sein (vgl. Hand 2018, S. 78). Jedenfalls geht Hand nicht weiter der Frage nach, ob es denn auch problematische Ausprägungen von moral formation geben kann, und, falls ja, woran diese zu erkennen wären. Demgegenüber wäre mit Ben Kotzee daran zu erinnern: „How moral attitudes are fostered is not not a worry“ (Kotzee 2020, S. 236; Hv.i.O.).

So dürfte zumindest eine problematische Ausprägung von Charaktererziehung darin bestehen, dass diese nicht oder zumindest nicht auch als eine Unterstützung der Entwicklung solcher Haltungen gefasst wird, die über Einsicht und eigenes Urteil vermittelt sind. Von einer Charaktererziehung in diesem Sinne ist in Hands Theorieentwurf jedoch nicht die Rede. Vielmehr droht moral formation zu einer konditionierenden Haltungserziehung zu avancieren, die insbesondere mit Vorschriften sowie Belohnung und Bestrafung für gezeigtes bzw. nichtgezeigtes Verhalten operiert (vgl. Hand 2018, S. 30 ff.). Damit aber wird der Anspruch unterlaufen, die Lebensform von Heranwachsenden nicht zu normieren, sondern diesen Mitwirkungsmöglichkeiten auch und vor allem in ihrer Charakterentwicklung zu ermöglichen.

Der Grund dafür, weshalb moral formation zu einer konditionierenden Haltungserziehung zu avancieren droht, scheint mir darin zu bestehen, dass Hand die Funktion der moral inquiry darauf reduziert, die Charaktererziehung der Heranwachsenden lediglich zu flankieren, denn ,the more often it occurs to me that I cannot justify my moral standards, the less steadfest I will become in holding myself and others to them“ (Hand 2018, S. 42). Hand erwägt hingegen nicht die Möglichkeit, dass es geboten sein könnte, moral inquiry der moral formation nicht (nur) zur Seite zu stellen, sondern dieser (auch) vorzuschalten, sollen die Haltungen, die Heranwachsende entwickeln, über Einsicht und eigenes Urteil vermittelt sein.

Knüpft man moralische Erziehung an den Anspruch, Heranwachsenden Mitwirkungsmöglichkeiten auch an der Entwicklung ihres Charakters zu ermöglichen, so ist es unzureichend, diese lediglich zu einem Handeln gemäß bestimmter moralischer Regeln anzuhalten, und diese damit auf eine vorgegebene moralische Ordnung hin festzulegen. In diesem Kontext hat Johann Friedrich Herbart die These formuliert, dass in der Erziehung dafür gesorgt werden müsse, in den Heranwachsenden eine „heilsame Charakterlosigkeit“ (Herbart 1964b [1806], S. 91) zu erhalten, bis diese dazu in der Lage sind, im Lichte eigener Einsichten und Urteilen zu handeln und in 
diesem Sinne an der eigenen Charakterentwicklung mitzuwirken. Eine solche Forderung mag besonders dann irritieren, wenn man sich vor Augen führt, dass Herbart die Unterstützung der Entwicklung von Moralität als den Zweck von Erziehung und Moralität dabei als „Charakterstärke der Sittlichkeit“ (Herbart 1964b [1806], S. 90) bestimmt hat. Für Herbart war offensichtlich klar: Eine Erziehung des moralischen Charakters schließt paradoxerweise ein, Charakterentwicklung nicht von Anfang an anzustreben, sondern diese vielmehr aufzuschieben, und Heranwachsenden damit die Möglichkeit offen zu halten, an der Entwicklung ihres Charakters mitzuwirken.

Aus der Warte von Hands Theorieentwurf betrachtet, könnte an dieser Stelle eingewendet werden, dass es doch naiv sei, zu glauben, man könne mit der Charaktererziehung warten, bis Heranwachsende zu einem Handeln im Lichte eigener Einsichten und Urteile aufgefordert werden können. Schließlich würden Heranwachsende sich selbst, aber auch Anderen durch uneinsichtiges Handeln oftmals Schaden zufügen, wenn sie nicht frühzeitig lernen, sich im Handeln auf elementare moralische Regeln zu verpflichten. Kurzum: Auf moral formation kann nicht verzichtet werden.

Herbart war freilich klar, dass Erziehung dem Umstand Rechnung tragen müsse, dass Heranwachsende durch uneinsichtiges Handeln sich selbst oder anderen Menschen schaden könnten. „Eine Erziehung, die sich um die Unordnungen der Kinder nicht bekümmerte, würde die Kinder selbst nicht kennen“ (Herbart 1964b [1806], S. 17). Sein Lösungsvorschlag besteht aber nicht darin, dass Heranwachsende bereits frühzeitig in ihrem Charakter normiert werden sollten. Der Vorschlag lautet vielmehr, dass Kinder regiert werden müssten. Eine regierende Erziehung ist darauf gerichtet, dass Heranwachsende an ihrem Handeln gehindert werden, um etwaigen Schaden zu verhindern. Zugespitzt formuliert: Der einzig legitime Zweck der Regierung ist es, ,,keinen Zweck im Gemüthe des Kindes zu erreichen“ (Herbart 1964b [1806], S. 19). Dies ist nur konsequent, wenn eine Erziehung zur Moralität den Anspruch impliziert, Heranwachsenden Mitwirkungsmöglichkeiten an der Entwicklung ihres Charakters offen zu halten. In diesem Fall bedarf es u. a. einer Form von Erziehung, die dem Umstand Rechnung trägt, dass Heranwachsende - gerade weil sie noch keine ,Charakterstärke der Sittlichkeit' entwickelt haben - in moralischer Hinsicht problematische Handlungen vollziehen, und die Heranwachsende gleichwohl nicht auf eine moralische Ordnung hin festlegt.

Den zweiten Einwand, der gegenüber Hands Theorieentwurf vorgebracht werden kann, bezeichne ich als den Einwand aus der Imperfektheit der Ethik. Um diesen Einwand zu entwickeln, ist es hilfreich, noch einmal kurz den Kern von Hands Entwurf in Erinnerung zu rufen. Heranwachsende dazu zu erziehen, moralische Regeln für gerechtfertigt zu halten, die vernünftigerweise umstritten sind, bedeutet nach Hand Indoktrination. Die Vermeidung von Indoktrination setzt entweder eine ,hieb- und stichfeste' Begründung von moralischen Regeln voraus, oder verlangt danach, moralische Fragen nichtdirektiv, d.h. mit offenem Ausgang zu thematisieren. Hands Theorieentwurf offeriert ein kontraktualistisches Argument zur Rechtfertigung bestimmter moralischer Regeln. Diese werden gleichsam als das Ergebnis eines Vertragsschlusses erläutert: Um unser gemeinsames Interesse an einem möglichst konfliktfreien Zusammenleben sicherzustellen, verpflichten wir uns gegenseitig zur Einhaltung bestimmter moralischer Ansprüche. 
An dieser Stelle ergibt sich nun das folgende Problem: Der Kontraktualismus bildet in der Ethik nur eine Position neben anderen. Es dürfte nicht zu bestreiten sein, dass es in der Ethik gut begründete Alternativen zum Kontraktualismus im Allgemeinen, aber auch zum Kontraktualismus à la Hand im Besonderen gibt. Die von Hand bemühte vertragstheoretische Argumentationsfigur muss bei genauer Betrachtung selbst als eine spezifische Variante innerhalb der Vertragstheorie verstanden werden. So argumentiert Hand aus der Warte einer Position, die moralische Regeln letztlich in Bezug auf das Eigeninteresse zu rechtfertigen versucht. Dem steht eine Position gegenüber, in der die Geltung moralischer Ansprüche aus der Warte eines transpersonalen, unparteilichen Beobachterstandpunkts rekonstruiert wird (vgl. Clayton und Stevens 2019, S. $511 \mathrm{f}$.).

Um nicht missverstanden zu werden: Ich möchte an dieser Stelle nicht für die These argumentieren, Hand hätte in seiner Theorie der moralischen Erziehung auf die ,falsche "Ethik rekurriert. Worum es mir geht, ist die schlichte Beobachtung, dass es sehr wohl vernünftige Alternativen zum Kontraktualismus à la Hand gibt. Diese Beobachtung allein ist ausreichend, um den Einwand zu formulieren, dass Hand in seiner Beschreibung von moralischer Erziehung eine spezifische Komplexität nicht berücksichtigt und hieraus die entsprechenden Konsequenzen gezogen hat - nämlich die Komplexität der Ethik.

Wir haben es in der Ethik mit der Situation eines vernünftigen Pluralismus zu tun. Das ,ethische Allgemeine“, so Jürgen Oelkers, lasse sich ,nicht wirklich allgemein bestimmen“, sondern immer nur in Abhängigkeit von spezifischen Voraussetzungen, die selbst wiederum strittig sind. ,Alle Theorien kommen unter dem Gesichtspunkt von Rivalität und Konkurrenz zustande, und keine Theorie bearbeitet das Allgemeine abschliessend" (Oelkers 1992, S. 12; Hv.i. O.). Die Ethik ist offenbar noch nicht fertig in dem Sinne, dass man die allein ,richtige' Begründung moralischer Ansprüche herausgefunden hätte. Die Ethik ist, wie man auch sagen könnte, imperfekt, d.h. noch nicht an ein Ende gekommen - sofern es überhaupt sinnvoll ist, ein definitives Ende der Ethik als möglich anzunehmen. Es gibt bis heute ein Ringen um die ,richtige' Ethik. Zugespitzt formuliert: Wir sind in modernen Gesellschaften nicht nur „moralisch unterwegs“ (Nöstlinger 1980), sondern befinden uns auch in der Ethik auf einer Suche nach Orientierung (vgl. Tugendhat 1993, S. 23 ff.). Hieraus folgt: Hands Rechtfertigungsversuch elementarer moralischer Regeln genügt dem eigenen Anspruch, ein Argument mit absoluter Durchschlagskraft bereitzustellen, nicht. Dieser Umstand bringt Hands Theorieentwurf in eine unliebsame Lage. Wendet man nämlich Hands Begriff der Indoktrination auf seinen Theorieentwurf selbst an, so kann man die von ihm beschriebene Erziehung als einer Kombination von moral formation und directive moral inquiry selbst als einen Indoktrinationsversuch der Heranwachsenden deuten. Zugespitzt formuliert: Hand perpetuiert mit seinem Theorieentwurf genau jenes Problem, für das er eigentlich eine Lösung offerieren wollte.

Auf diese Problematik ist in der Debatte um Hands Theorieentwurf bereits mehrfach hingewiesen worden. So haben Clayton und Stevens aus der Einsicht, dass „contractarianism is itself subject to reasonable disagreement“, den Schluss gezogen, ,that to teach contractarianism and what follows from it directively would count as indoctrination, on Hand's view“" (Clayton und Stevens 2019, S. 504). Der 
von mir formulierte Einwand ist insofern nicht gänzlich neu. Jedoch sind aus dieser Einsicht bislang nicht die erforderlichen Konsequenzen gezogen worden. Eine solche Konsequenz wäre darin zu sehen, dass die Annahme aufgegeben werden muss, Halt in einer vermeintlich definitiven Ethik finden zu können. An dieser Stelle rückt die Theorie nichtaffirmativer Moralerziehung als eine attraktive Alternative in den Blick, wird in dieser Theorie doch von der Voraussetzung ausgegangen, dass es weder eine definitive Moral noch die eine ,richtige“ Ethik gebe, und das Problem, wie moralische Erziehung unter den Bedingungen einer imperfekten Moral und Ethik bestimmt werden kann, in den Mittelpunkt der Theoriebildung gerückt (vgl. Benner 2006, S. 187f.).

\section{Die Alternative}

Die Theorie nichtaffirmativer Moralerziehung, wie sie insbesondere von Dietrich Benner in der Auseinandersetzung mit der Tradition moderner Pädagogik entwickelt worden ist, wird explizit mit dem Anspruch verknüpft, eine Beschreibung von Moralerziehung anzufertigen, in der dem Umstand Rechnung getragen wird, dass das Aufwachsen in modernen Gesellschaften ,unter Bedingungen einander widerstreitender Moralen“ (Benner 2007, S. 182) seinen Ort hat. Als grundlegend erweist sich in diesem Zusammenhang die Differenz zwischen einer affirmativen und einer nichtaffirmativen Moralerziehung.

Affirmative Moralerziehung meint eine Form des Miteinanderumgehens, in der Heranwachsende in eine bestimmte Moral eingeführt werden, die diese übernehmen und an der sie ihr Handeln orientieren sollen. Erziehung avanciert in diesem Sinne zu einer „Weitergabe positiver Unterscheidungen zwischen einem vorbestimmten Guten und einem vordefinierten Bösen“ (Benner 2007, S. 176). Die Voraussetzung, auf der eine solche Beschreibung von moralischer Erziehung beruht, lautet, dass das moralisch Gebotene und Verbotene sowie das moralisch Neutrale zweifelsfrei feststehen, so dass es letztlich keinen plausiblen Grund dafür gibt, die entsprechenden moralischen Regeln nicht an die Heranwachsenden zu tradieren. Demgegenüber bedeutet nichtaffirmative Moralerziehung eine Form des Miteinanderumgehens, in der Heranwachsenden dabei geholfen wird, die Fähigkeit und Bereitschaft zu entwickeln, ,,in moralischen Fragen selbst zu urteilen und im Handeln eigenen Einsichten zu folgen“ (Benner 2007, S. 170). Nichtaffirmative Moralerziehung in diesem Sinne ist im Unterschied zu Spielarten einer affirmativen Moralerziehung darauf gerichtet, auf der Seite der Heranwachsenden eine ,Zivilcourage“ zu befördern, die auf „eigener Urteilskraft“ gründet (Benner 2007, S. 175) und in einer Selbstverpflichtung auf überindividuelle Verbindlichkeiten zum Ausdruck kommt.

Eine solche Beschreibung von moralischer Erziehung setzt voraus, dass die Frage, was wir uns wechselseitig als Menschen schulden, als eine solche begriffen wird, die nicht schon vor aller Erziehung ihre Beantwortung erfahren hat, so dass Erziehung nur mehr bereits gefundene Antworten zu tradieren hätte. Nichtaffirmative Moralerziehung tradiert das Problem der Moral vielmehr als Frage, d.h. sie ist darauf gerichtet, die Heranwachsenden in die Suche nach dem Guten und Bösen selbst hineinzuziehen. Moralerziehung in diesem Sinne bedeutet gerade nicht, 
die Frage, was wir uns wechselseitig als Menschen schulden, stellvertretend für die Heranwachsenden zu beantworten, sondern diese so in diese Frage einzuführen, dass sie die Voraussetzungen entwickeln, um an moralischen Diskursen urteilsfähig zu partizipieren. Dies hat u.a. zur Konsequenz, dass moralische Ansprüche an die Heranwachsenden in der Erziehung nicht einfach durchgesetzt werden dürfen, sondern vielmehr in „diskursive Aufgaben“ transformiert werden müssen, so dass sie zu „Gegenständen einer intergenerationellen Verständigung“ werden können. „Eine solche Verständigung entwickelt die Regeln des Zusammenlebens denkend, urteilend und experimentierend gemeinsam mit den Lernenden“, und zwar auch dadurch, dass sie moralische Ansprüche unter Berücksichtigung von „Erfahrungen“, die Heranwachsenden mit diesen machen, ,problematisiert und neu aushandelt" (Benner 2015, S. 111). ${ }^{3}$

Nichtaffirmative Moralerziehung nimmt ihren Ausgang zwar von vorgegebenen Moralen, affirmiert diese jedoch nicht, sondern behandelt sie als Bestimmungen, die Menschen auf ihrer Suche nach Orientierung eines gelingenden Lebens und Zusammenlebens hervorgebracht haben, und die gerade deshalb, weil Menschen imperfekte Lebewesen sind, zukünftig erneut zu Gegenständen und Ausgangspunkten der Wechselwirkung von Mensch und Welt werden können. Dies schließt moralische Festlegungen, die Menschen in ihrem Aufwachsen unter bestimmten sozialen Bedingungen bereits entwickelt haben, ein. Um Heranwachsenden die Möglichkeit zu eröffnen, sich zu entsprechenden Festlegungen in ein Verhältnis zu setzen, suchen pädagogische Akteure Unterbrechungen im Urteilen und Handeln von Heranwachsenden zu provozieren. Solche edukativ veranlassten ,negativen Erfahrungen im Bereich der Moral“ (Benner 2006, S. 187) erfüllen nicht die Funktion, eine bestimmte Moral durchzusetzen. Die Differenzerfahrungen, die im Kontext einer nichtaffirmativen Moralerziehung anvisiert werden, sind vielmehr dadurch gekennzeichnet, dass sie eine „Verarbeitung“ zulassen und auch verlangen, die ,auf ein Selbst-Denken, -Urteilen und -Handeln bezogen ist“" (Benner 2006, S. 187). Insofern ist eine nichtaffirmative Moralerziehung nicht darauf gerichtet, Heranwachsende in eine ,festgefügte Moral“ (Benner 2006, S. 187) einzuführen, sondern vielmehr darauf bezogen, Heranwachsende mit moralischen Ansprüchen zu konfrontieren, und diesen dabei zu helfen, erlittene Differenzerfahrungen durchzuarbeiten - und zwar so, dass Heranwachsende zu einem ,Experimentieren innerhalb und mit den gesellschaftlich vorgegebenen Moralen“ (Benner 2006, S. 187) aufgefordert werden. Moralische Erziehung in diesem Sinne geht nicht darin auf, dass pädagogische Akteure Heranwachsenden die für sie maßgeblichen Moralen präsentieren - was ohnehin unvermeidlich ist -, sondern impliziert, dass moralische Ansprüche unter Angabe von Gründen gerechtfertigt werden. Nichtaffirmative Moralerziehung bedeutet darüber hinaus, alternative Moralen zu repräsentieren, um Heranwachsenden die Möglichkeit zu eröffnen, Blickwechsel zu vollziehen, d.h. bereits entwickelte

\footnotetext{
3 Moralische Regeln in ,diskursive Aufgaben` zu überführen, dient dem Zweck, Heranwachsenden Möglichkeiten des Sich-Verhaltens in Fragen des moralisch Gebotenen, Verbotenen und Neutralen zu eröffnen. Eine Beschreibung nichtaffirmativer Moralerziehung beruht hingegen nicht auf der darüber hinausgehenden diskursethischen Prämisse, dass über die Geltung von moralischen Ansprüchen allein diskursiv entschieden werden könne.
} 
moralische Festlegungen zu transzendieren. Schließlich ist eine Erziehung zur Moralität darauf gerichtet, Heranwachsende dabei zu begleiten, eigenen moralischen Positionsbestimmungen im Handeln zu entsprechen (vgl. Mertens 1999, S. 39 f.).

Moralische Erziehung in diesem Sinne als Einführung in den Widerstreit zwischen verschiedenen Moralen und diese stützende Ethiken zu konzipieren, hat den Vorteil, dass der Vorwurf der Indoktrination nicht auf die Beschreibung selbst zurückfällt. Nun könnte an dieser Stelle aus der Warte von Hands Theorieentwurf kritisch rückgefragt werden, ob es tatsächlich überzeugend ist, sämtliche moralische Ansprüche dem Experimentieren anheim zu stellen. Wird damit nicht dem Relativismus Tür und Tor geöffnet? Mit dieser Frage kann ein Problem in den Blick gerückt werden, das in der Theorie nichtaffirmativer Moralerziehung bislang nicht die ihm gebührende Aufmerksamkeit erfahren hat. Es stellt sich nämlich in der Tat die Frage, ob es bei allem Widerstreit zwischen verschiedenen Moralen und Ethiken nicht doch invariante Bezugsgrößen gibt, die auch im Kontext einer nichtaffirmativen Erziehung direktiv, d.h. mit Überzeugungsabsicht thematisiert werden sollten.

In der Theorie nichtaffirmativer Moralerziehung wird von der Prämisse ausgegangen, dass die „Freiheit der Wahl einer eigenen Lebensform, auf die Heranwachsende durch Erziehung vorbereitet werden“ sollen, die „Freiheit der Wahl einer persönlichen Moral“ impliziere (Benner 2006, S. 179). In diesem Votum für die Freiheit der Heranwachsenden scheint mir der entscheidende Grund dafür zu liegen, Moralerziehung überhaupt als nichtaffirmativ zu konzipieren. Die Freiheit der Wahl einer persönlichen Moral dürfe jedoch, so Benner, nicht im Sinne eines ,,beliebigen Dezisionismus“ verstanden werden, sondern müsse vielmehr als eine „Freiheit“ begriffen werden, die ,die Achtung der Freiheit der Anderen, an der Hervorbringung und Interpretation von Moral mitzuwirken, einschließt" (Benner 2006, S. 179). Damit ist zugleich gesagt, dass im Kontext einer nichtaffirmativen Moralerziehung nicht allen Moralen der gleiche Stellenwert zugesprochen werden kann, gibt es doch moralische Positionsbestimmungen, die mit dem Anspruch der, Achtung der Freiheit der Anderen“ inkompatibel sind. An dieser Stelle setzen die Überlegungen zur Weiterentwicklung der Theorie nichtaffirmativer Moralerziehung an, die ich im Folgenden zur Diskussion stellen möchte.

\section{Differenzierungen}

Die erste Überlegung betrifft das Problem der Rechtfertigung der moralischen Grundorientierung, die im Kontext dieser Theorie als verbindlich vorausgesetzt wird. Benner thematisiert diese Rahmung insbesondere im Rückgriff auf die praktischen Ideen der inneren Freiheit, der Vollkommenheit, des Wohlwollens, des Rechts und der Billigkeit, wie sie von Herbart in der Allgemeinen praktischen Philosophie bestimmt worden sind (vgl. Benner 2006, S. $182 \mathrm{ff}$.). Diesem Vorschlag liegt u. a. die Annahme zugrunde, dass diese Ideen als ein Versuch interpretiert werden können, den in Kants kategorischem Imperativ formulierten Anspruch der wechselseitigen Achtung relational zu verschiedenen Konstellationen menschlichen Lebens und Zusammenlebens auszulegen. Ob eine solche Interpretation überzeugend ist, sei an dieser Stelle dahingestellt. Entscheidend ist, dass das Experimentieren mit vorge- 
gebenen oder stellvertretend antizipierten Moralen und Ethiken in einem Rahmen situiert wird, der selbst nicht zur Debatte zu stehen scheint. Andrea Thimm hat deshalb die Frage aufgeworfen, ob und, falls ja, wie es eigentlich gerechtfertigt werden kann, die praktischen Ideen Herbarts als maßgeblichen Rahmen experimenteller Moralerziehung in Anschlag zu bringen, erweist sich eine solche ,Eingrenzung des Experimentierfeldes“ doch als ,ziemlich voraussetzungsvoll und nicht selbstverständlich“ (Thimm 2007, S. 156). Dies ist insbesondere dann der Fall, wenn die Problemstellung lautet, eine Beschreibung von moralischer Erziehung anzufertigen, die ohne Ausgriff auf eine vermeintlich definitive Ethik auskommt.

In der Theorie nichtaffirmativer Erziehung ist der Anspruch maßgeblich, dass Erziehung die Lebensform von Heranwachsenden - auch in Sachen Moral - nicht normieren dürfe, sondern diese vielmehr für ein Leben in Selbstbestimmung freisetzen müsse. Ein experimenteller Umgang mit Moral jenseits der Erziehung setzt jedoch voraus, dass Menschen sich auch über die Erziehung hinaus wechselseitig Spielräume für einen entsprechenden Umgang zugestehen bzw. allererst eröffnen. Die Freiheit, an der Bildung der Moral mitzuwirken, muss im Miteinanderumgehen der Menschen - auch immer wieder neu - hergestellt und stabilisiert werden. Hieraus folgt, dass eine nichtaffirmative Moralerziehung nicht allein darin bestehen kann, Heranwachsende darin zu unterstützen, mit verschiedenen Moralen zu experimentieren. Eine nichtaffirmative Moralerziehung wird darüber hinaus auch dafür Sorge tragen müssen, dass Heranwachsende eine Haltung entwickeln, die in einer Achtung der Freiheit anderer Menschen, ihre Lebensform zu wählen, zum Ausdruck kommt (vgl. Schaber 2010, S. $145 \mathrm{ff}$.).

Es wäre widersinnig, den Anspruch, Menschen in ihrer Freiheit zu achten, nicht als einen verbindlichen Anspruch an die Heranwachsenden heranzutragen, dem diese entsprechen lernen sollen. Man müsste dann nämlich erklären, warum Erziehung überhaupt als eine Form des Miteinanderumgehens konzipiert werden sollte, in der Heranwachsenden ein experimenteller Umgang mit Moral ermöglicht wird, wenn man zugleich dazu bereit ist, diesen Anspruch zu relativieren, indem im Kontext einer nichtaffirmativen Erziehung Positionen als gleichberechtigt behandelt werden, die mit einer solchen Orientierung inkompatibel sind. Der Anspruch, dass Menschen sich wechselseitig in ihrer Freiheit achten sollen, eigene Positionen zu entwickeln, zu leben, zu kritisieren, zu verteidigen, zu verwerfen und weiterzuentwickeln, kann im Kontext nichtaffirmativer Moralerziehung nicht sinnvoll dem Experimentieren der Heranwachsenden anheimgestellt werden, ohne damit den Vorschlag, moralische Erziehung überhaupt als nichtaffirmativ zu begreifen, selbst ad absurdum zu führen. Im Kontext nichtaffirmativer Moralerziehung kann z. B. sinnvoll mit offenem Ausgang darüber diskutiert werden, ob es moralisch geboten sei, sich vegetarisch zu ernähren. Nicht aber kann in diesem Rahmen sinnvoll nichtdirektiv darüber diskutiert werden, ob es Menschen zusteht, zur Frage einer moralisch gerechtfertigten bzw. ungerechtfertigten Ernährungsweise Stellung zu nehmen.

Nichtaffirmative Moralerziehung wäre so gesehen nicht konsequent zu Ende gedacht, wenn sie nicht zugleich als Konfrontation mit einer moralischen Grundorientierung bestimmt werden würde, die dem experimentellen Umgang von Heranwachsenden mit Moralen Grenzen zieht. Diese Bezugsgröße - und das ist hier entscheidend - besitzt nicht deshalb einen besonderen Status, weil einer spezifischen 
Ethik ein Primat zugesprochen wird. Der Grund hierfür ist vielmehr darin zu sehen, dass die wechselseitige Achtung von Freiheit gleichsam als die Voraussetzung eines Zusammenlebens in Pluralität fungiert. Der Anspruch, Heranwachsende für ein Leben in Selbstbestimmung freizusetzen, korreliert in diesem Sinne immer schon mit einer zweiten Aufgabe, nämlich dafür Sorge zu tragen, dass Heranwachsende lernen, auch anderen Menschen die Freiheit zuzugestehen bzw. allererst zu eröffnen, ihre Lebensform zu wählen und - damit verbunden - eine persönliche Moral zu entwickeln.

Diese Überlegung hat nicht zur Konsequenz, dass nichtaffirmative Moralerziehung an dieser Stelle in affirmative Moralerziehung umschlägt. Moralische Ansprüche mit Überzeugungsabsicht an die Heranwachsenden zu adressieren, ist nicht gleichbedeutend damit, Heranwachsende unbefragt auf die jeweiligen Ansprüche festzulegen. Sollten die vorangegangenen Überlegungen triftig sein, so bedeutet dies, dass es auch im Kontext einer nichtaffirmativen Erziehung moralische Ansprüche gibt, die sinnvoll nur in Form einer direktiven Moralerziehung thematisiert werden können. Hand bezeichnet damit eine Form des Miteinanderumgehens, in der Heranwachsende dazu bewogen werden sollen, bestimmte moralische Regeln für gerechtfertigt zu halten. Mein Vorschlag lautet, dem Ausdruck ,direktive Erziehung' im Kontext einer Theorie nichtaffirmativer Moralerziehung eine spezifischere Bedeutung zu geben und hierunter eine Ausrichtung von Erziehung zu verstehen, die darauf bezogen ist, Heranwachsende darin zu unterstützen, Einsicht in nicht sinnvoll bestreitbare moralische Ansprüche zu gewinnen. Von einer direktiven nichtaffirmativen Moralerziehung wäre eine Ausrichtung zu unterscheiden, die als nichtdirektiv bezeichnet werden kann, und die darin besteht, dass Heranwachsende zu einem experimentellen Umgang mit differenten Positionen aufgefordert werden, um unter den Bedingungen moralischen Widerstreits eigene moralische Urteile $\mathrm{zu}$ entwerfen und diesen im Handeln zu entsprechen.

Berücksichtigt man die Unterscheidung zwischen diesen beiden Ausrichtungen nichtaffirmativer Moralerziehung, so wäre es verfehlt, wollte man diese vorschnell auf eine experimentelle Moralerziehung reduzieren. Von dieser sollte vielmehr nur dann gesprochen werden, wenn nichtaffirmative Moralerziehung nichtdirektiv ausgerichtet ist. Umgekehrt wäre es ebenso verfehlt, nichtaffirmative und direktive Erziehung für inkompatibel zu erklären. Nichtaffirmative Moralerziehung ist durchaus kompatibel damit, dass bestimmte moralische Ansprüche mit Überzeugungsabsicht thematisiert werden - dies jedenfalls dann, wenn Heranwachsende zu einer Prüfung von Geltungsansprüchen aufgefordert werden, so dass diese die Möglichkeit haben, die jeweiligen Ansprüche einzusehen und sich damit in Freiheit auf überindividuelle Verbindlichkeiten zu verpflichten.

Die zweite Überlegung zur Weiterentwicklung der Theorie nichtaffirmativer Moralerziehung betrifft die Frage, welche Grundformen einer Erziehung zur Moralität unterschieden, und wie diese so relationiert werden können, dass das Kombinationsdefizit, das in Hands Theorieentwurf ausgemacht worden ist, vermieden wird. Der Vorschlag sieht eine Unterscheidung zwischen einer regierenden Erziehung, einer Erziehung durch Unterricht und einer beratenden Erziehung vor (vgl. Benner 2015, S. 216 ff.; Herbart 1964b [1806]), wobei diese Grundformen jeweils im Lichte der Theorie nichtaffirmativer Moralerziehung ausgelegt werden. 
Ich bin in der Problematisierung von Hands Theorieentwurf bereits auf die Regierung zu sprechen gekommen, so dass ich mich an dieser Stelle kurz fassen kann. Entscheidend ist, dass eine regierende Erziehung darauf gerichtet ist, Schaden zu verhindern, den Heranwachsende erleiden bzw. den diese sich selbst oder Anderen und Anderem zufügen könnten, würden diese nicht vom einem bestimmten Handeln abgehalten werden. Regierung in diesem Sinne bedeutet gerade nicht, Heranwachsende zu bestimmten Haltungen zu bewegen, indem Regeln vorgeschrieben sowie regelkonformes Verhalten belohnt und regelwidriges Verhalten bestraft werden. Die Maßgabe lautet vielmehr, die Entstehung von Charakterzügen erst einmal nicht zu forcieren, um Heranwachsende in ihrer Lebensform nicht vorschnell festzulegen, sondern diesen die Möglichkeit offenzuhalten, an der Entwicklung ihres Charakters mitzuwirken. Zugleich wird man davon ausgehen müssen, dass Heranwachsende - gerade weil sie noch keinen moralischen Charakter entwickelt haben - Handlungen vollziehen, die sich im Lichte der Idee wechselseitiger Achtung von Freiheit als problematisch erweisen und die - sofern sie regelmäßig vollzogen werden - dazu beitragen könnten, das Charakterzüge entstehen, die moralisch ebenfalls zu missbilligen wären. Aus diesem Grund kann auf eine regierende Erziehung nicht verzichtet werden.

Im Unterschied zur Regierung, die Heranwachsende am Handeln hindert, ist der Unterricht darauf gerichtet, diese zu einem Wissen zu führen, das im alltäglichen Miteinanderumgehen nicht angeeignet werden kann. Unterricht erweist sich allein schon deshalb als ein unverzichtbares Moment einer nichtaffirmativen Moralerziehung, weil ein entsprechendes Wissen erforderlich ist, damit Heranwachsende bestimmte Sachverhalte überhaupt moralisch beurteilen sowie den jeweiligen Urteilen handelnd entsprechen können. Wer nicht weiß, dass ein Großteil der Treibhausgasemissionen aus der Haltung von Tieren resultiert, wird kaum dazu in der Lage sein, zu bestimmten Ernährungsfragen in moralischer Hinsicht Stellung zu nehmen. Wer nicht fähig ist, einen Brief zu verfassen, wird seiner Empörung in der Lokalzeitung nur schwer Ausdruck verschaffen können.

Sachliche Einsichten sind zunächst einmal indifferent hinsichtlich der Lebensführung von Heranwachsenden im Umgang mit Anderen. Damit diese Einsichten handlungsrelevant werden können, bedarf es eines Bindeglieds, das als Werturteil bezeichnet werden kann. Im Werten, d. h. im Fällen von Werturteilen antworten Heranwachsende auf die Frage nach der Bedeutung einer sachlichen Einsicht für ein gelingendes Leben und Zusammenleben. Ein Unterricht, in dem dieser Umstand Berücksichtigung findet, wird traditionell als erziehender Unterricht bezeichnet (vgl. Rucker 2019). Ein erziehender Unterricht ist dadurch gekennzeichnet, dass Heranwachsende dazu aufgefordert werden, im Lichte sachlicher Einsichten zu Fragen eines gelingenden Lebens und Zusammenlebens Stellung zu nehmen, ihre Positionsbestimmungen zu begründen sowie diese der Konfrontation mit Alternativen auszusetzen. In diesem Zusammenhang besteht für die Heranwachsenden die Möglichkeit, mit verschiedenen Moralen und Ethiken zu experimentieren. Ein erziehender Unterricht ist nämlich nicht darauf gerichtet, Heranwachsende in einer bestimmten Moral oder Ethik zu unterweisen, sondern vielmehr darauf bezogen, moralische und ethische Positionen zu thematisieren, d.h. zu interpretieren, zu befragen und - unter Umständen - zu problematisieren, und damit zugleich in die Schwierigkeiten einzu- 
führen, eine allgemein verbindliche Moral und Ethik zu finden und zu begründen. Eine kontraktualistische Rechtfertigung elementarer moralischer Regeln à la Hand avanciert unter diesen Bedingungen zu einer Position neben anderen, die auf ihre Möglichkeiten und Grenzen hin bedacht werden.

Dies bedeutet nicht, dass Fragen, in denen verschiedene Moralen miteinander konkurrieren, immer als kontrovers, d.h. mit offenem Ausgang thematisiert werden sollten. Nicht zur Debatte steht in einem erziehenden Unterricht die für eine nichtaffirmative Moralerziehung maßgebliche Grundorientierung, allen Menschen die gleiche Freiheit zuzugestehen bzw. allererst zu eröffnen, eine eigene Lebensform $\mathrm{zu}$ suchen, $\mathrm{zu}$ finden und zu leben. Im Unterschied zu einer regierenden Erziehung, in der diese Grundorientierung durchgesetzt wird, kommt dem Unterricht die Aufgabe zu, Heranwachsenden Einsicht in die Verbindlichkeit dieser Grundorientierung zu ermöglichen. Dies impliziert, Urteile von Heranwachsenden, in denen diese Grundorientierung missachtet wird, nicht unbefragt zu akzeptieren. Von einem erziehenden Unterricht wäre vielmehr zu erwarten, dass moralisch verwerfliche Positionsbestimmungen thematisiert und Heranwachsende darin unterstützt werden, einzusehen, dass und warum diese nicht akzeptiert werden können. Erziehender Unterricht bedeutet dann, Heranwachsenden dabei zu helfen, sich von bereits entwickelten moralischen Festlegungen im Lichte besser begründeter Alternativen zu emanzipieren.

Vom Unterricht ist schließlich eine beratende Erziehung zu unterscheiden, deren Aufgabe darin besteht, Heranwachsenden dabei zu helfen, im Lichte eigener Einsichten und Urteile zu handeln, d.h. sie in konkreten Fragen der eigenen Lebensführung im Umgang mit Anderen zu unterstützen. Im Kontext einer Erziehung zur Moralität bedeutet dies vor allem, dafür Sorge zu tragen, dass Heranwachsende im Handeln nicht unmittelbar ihrem Willen folgen, sondern dazu veranlasst werden, ihren Willen daraufhin zu prüfen, ob dieser mit moralischen Einsichten und eigenen moralischen Urteilen kompatibel ist.

Grundsätzlich ist davon auszugehen, dass allein schon der Umstand, dass pädagogische Akteure auf bestimmte Art und Weise mit den Heranwachsenden umgehen, für die Entwicklung von deren Charakter nicht folgenlos bleibt. Vor diesem Hintergrund dürfte für eine Entwicklung der Haltung, Menschen als Freiheitswesen zu achten, gerade die Erfahrung von zentraler Bedeutung sein, in der Erziehung selbst als Freiheitswesen anerkannt und entsprechend adressiert zu werden. Heranwachsenden in der Erziehung Mitwirkungsmöglichkeiten zu eröffnen, trägt so gesehen nicht nur dazu bei, dass diese sich selbst als Subjekte begreifen lernen, sondern erweist sich darüber hinaus als bedeutsam dafür, dass diese eine Haltung entwickeln, ihrerseits andere Menschen als Subjekte zu achten. „Die Anerkennung des Edukanden in seiner Egalität ist ein wesentlicher Bestandteil der moralischen Erziehung. Nur derjenige, der als Mensch anerkannt wird, ist auch fähig, andere anzuerkennen“ (Herzog 1991, S. 59). Dies bedeutet freilich nicht, dass Heranwachsende die Achtung, die sie im Miteinanderumgehen erfahren, bereits reflexiv einholen könnten, und es bedeutet auch nicht, dass Heranwachsende allein schon aufgrund der ihnen entgegengebrachten Achtung die Fähigkeit entwickeln, spezifische Sachverhalte moralisch zu beurteilen sowie den eigenen Urteilen im Handeln zu entsprechen. Es ist gerade diese „Bewältigung der Komplexität ihres Lebens“ (Herzog 1991, S. 59), zu 
der Heranwachsende auf Unterstützung angewiesen sind, und es ist diese Aufgabe, die im Mittelpunkt einer beratenden Erziehung steht.

Eine entsprechende Hilfe ist nicht nur deshalb erforderlich, weil in konkreten Situationen nicht immer auf der Hand liegt, was moralisch geboten, verboten oder neutral ist, sondern dieses allererst ermessen werden muss. Nicht nur um die Entwicklung von Urteilskraft zu unterstützen, bedarf es einer beratenden Erziehung, sondern auch deshalb, weil nicht vorausgesetzt werden kann, dass bereits entwickelte Charakterzüge sich stets als kompatibel mit den Handlungsentwürfen von Heranwachsenden erweisen. Darüber hinaus wäre zu berücksichtigen, dass ein Handeln, das an moralischen Ansprüchen orientiert ist, mit einer Einschränkung des Eigeninteresses einhergeht. Von daher muss davon ausgegangen werden, dass es in der Entwicklung eines moralischen Charakters - auch immer wieder neu - zu Situationen kommen wird, die existentielle Entscheidungen verlangen - Situationen, die von Herbart als ,innerer Kampf ‘ beschrieben worden sind. Dieser ,Kampf‘ muss siegreich bestritten werden - nicht nur, damit ein Entwurf zu einem entsprechenden Handeln führt, sondern auch, damit neue Charakterzüge entstehen können, die eine Achtung der Freiheit Anderer zum Ausdruck bringen (vgl. English 2013, S. 30 ff.). Von daher wäre es problematisch, Heranwachsenden entsprechende Differenzerfahrungen zu ersparen. Im Kontext einer beratenden Erziehung geht es deshalb immer auch darum, Heranwachsende in Situationen der Wahl hineinzuziehen und ihnen dabei zu helfen, im Lichte möglicher Alternativen eigene Entscheidungen zu treffen sowie den Entschluss in Handlung umzusetzen. Hierbei kommt es maßgeblich auf die einzelne Person an, die vor der Aufgabe steht, das Eigeninteresse zurückzustellen und im Handeln einem moralischen Anspruch zu folgen. Für pädagogische Akteure bedeutet dies notwendigerweise ein Wagnis, denn es ist nicht vorhersehbar, wie Heranwachsende sich entscheiden werden (vgl. Biesta 2013, S. 2 ff.). Umgekehrt kommt eine Erziehung zur Moralität nicht daran vorbei, Heranwachsenden entsprechende Freiräume zu eröffnen, sollen diese die Möglichkeit haben, an der Entwicklung ihres Charakters mitzuwirken und sich in Freiheit auf überindividuelle Verbindlichkeiten zu verpflichten.

\section{Ein Fazit}

In diesem Beitrag habe ich zunächst versucht, den Problemraum einer Theorie der Erziehung zur Moralität in einer komplexen Welt zu umreißen. Hiervon ausgehend habe ich zwei Vorschläge, wie moralische Erziehung unter den Bedingungen von Komplexität sinnvoll konzipiert werden könnte, rekonstruiert und aufeinander bezogen. Die Konfrontation beider Beschreibungen von Moralerziehung hat zu zwei Erkenntnissen geführt: Zum einen konnte gezeigt werden, dass die Theorie nichtaffirmativer Moralerziehung spezifische Einwände, die gegenüber Hands Theorieentwurf vorgebracht werden können, zu vermeiden erlaubt. Zum anderen hat sich gezeigt, dass die Theorie nichtaffirmativer Moralerziehung in bestimmter Hinsicht weiterentwickelt, zumindest aber präzisiert werden muss. Vor diesem Hintergrund habe ich zunächst einen Vorschlag entwickelt, wie die moralische Grundorientierung, die in diesem Ansatz als verbindlich vorausgesetzt wird, begründet werden kann. 
Anschließend habe ich Überlegungen zur Diskussion gestellt, wie nichtaffirmative Moralerziehung als ein Zusammenhang dreier Grundformen bestimmt werden kann, für den sowohl eine direktive als auch eine nichtdirektive Ausrichtung maßgeblich ist. Der Vorschlag besteht darin, zwischen einer regierenden Erziehung, einer Erziehung durch Unterricht und einer beratenden Erziehung zu unterscheiden.

Herbart hatte bekanntlich die Auffassung vertreten, dass „Moralität“ als „höchster Zweck“ auch der „Erziehung“ unter den Menschen ,allgemein anerkannt“ sei. „Wer dies leugnete“, so Herbart, ,müßte wol nicht eigentlich wissen, was Moralität ist; wenigstens hätte er kein Recht hier mitzusprechen“ (Herbart 1964a [1804], S. 259). Im Lichte der hier zur Diskussion gestellten Überlegungen kann Herbarts Überlegung als ein Hinweis darauf interpretiert werden, dass eine Erziehung, die darauf gerichtet ist, Heranwachsenden dabei zu helfen, ihre Lebensform selbst zu bestimmen, zugleich mit einschließen muss, Heranwachsende darin zu unterstützen, einzusehen, dass eine solche Freisetzung für eine selbstbestimmte Lebensführung auf bestimmte soziale Voraussetzungen angewiesen ist. Aus diese Grund müssen Heranwachsende eine Haltung entwickeln, die in einer Achtung der Freiheit anderer Menschen zum Ausdruck kommt, ihre Lebensform zu wählen. ,Wer dies', d.h. diesen Zusammenhang von Selbstbestimmung und Moralität, leugnete‘, so könnte man mit Herbart formulieren, ,müßte wol nicht eigentlich wissen, was Moralität ist ‘, nämlich Voraussetzung dafür, dass ein Zusammenleben in Pluralität möglich ist.

Funding Open access funding provided by University of Bern

Open Access Dieser Artikel wird unter der Creative Commons Namensnennung 4.0 International Lizenz veröffentlicht, welche die Nutzung, Vervielfältigung, Bearbeitung, Verbreitung und Wiedergabe in jeglichem Medium und Format erlaubt, sofern Sie den/die ursprünglichen Autor(en) und die Quelle ordnungsgemäß nennen, einen Link zur Creative Commons Lizenz beifügen und angeben, ob Änderungen vorgenommen wurden.

Die in diesem Artikel enthaltenen Bilder und sonstiges Drittmaterial unterliegen ebenfalls der genannten Creative Commons Lizenz, sofern sich aus der Abbildungslegende nichts anderes ergibt. Sofern das betreffende Material nicht unter der genannten Creative Commons Lizenz steht und die betreffende Handlung nicht nach gesetzlichen Vorschriften erlaubt ist, ist für die oben aufgeführten Weiterverwendungen des Materials die Einwilligung des jeweiligen Rechteinhabers einzuholen.

Weitere Details zur Lizenz entnehmen Sie bitte der Lizenzinformation auf http://creativecommons.org/ licenses/by/4.0/deed.de.

Interessenkonflikt T. Rucker gibt an, dass kein Interessenkonflikt besteht.

\section{Literatur}

Benner, D. (2006). Moral und Bildung. Über ihr problematisches Verhältnis in der griechischen Polis und in der Bildungstheorie der Moderne. Vierteljahresschrift für wissenschaftliche Pädagogik, 82(2), $168-191$.

Benner, D. (2007). Moralische Erziehung und Bildung der Moral. In A. A. Bucher (Hrsg.), Moral, Religion, Politik: Psychologisch-pädagogische Zugänge (S. 169-183). Wien: LIT.

Benner, D. (2015). Allgemeine Pädagogik. Eine problemgeschichtlich-systematische Einführung in die Grundstruktur pädagogischen Denkens und Handelns (8. Aufl.). Weinheim: Beltz Juventa.

Biesta, G. (2013). The beautiful risk of education. Boulder: Paradigm Publishers.

Birnbacher, D. (2013). Moral and other values. Kultura I Wartosci, 4(8), 43-58. 
Clayton, M., \& Stevens, D. (2019). The poverty of contractarian moral education. Journal of Moral Education, 48(4), 501-514.

Copp, D. (2009). Towards a pluralist and teleological theory of normativity. Philosophical Issues, 19(1), 21-37.

Copp, D. (2016). Moral education versus indoctrination. Theory and Research in Education, 14(2), 149-167.

English, A. (2013). Discontinuity in learning. Dewey, Herbart and education as transformation. New York: Cambridge University Press.

Hand, M. (2007). Should we teach homosexuality as a controversial issue? Theory and Research in Education, 5(1), 69-86.

Hand, M. (2014). Towards a theory of moral education. Journal of Philosophy and Education, 48(4), $519-532$.

Hand, M. (2018). A theory of moral education. New York: Routledge.

Herbart, J.F. (1964a). Über die ästhetische Darstellung der Welt, als das Hauptgeschäft der Erziehung. In K. v. Kehrbach \& O. Flügel (Hrsg.), Joh. Fr. Herbart's Sämtliche Werke in chronologischer Reihenfolge (Bd. 1, S. 259-274). Aalen: Scientia.

Herbart, J.F. (1964b). Allgemeine Pädagogik aus dem Zweck der Erziehung abgeleitet. In K. v. Kehrbach \& O. Flügel (Hrsg.), Joh. Fr. Herbart's Sämtliche Werke in chronologischer Reihenfolge (Bd. 2, S. 1-139). Aalen: Scientia.

Herzog, W. (1991). Die Banalität des Guten. Zur Begründung der moralischen Erziehung. Zeitschrift für Pädagogik, 37(1), 41-64.

Koller, P. (1983). Rationalität und Moral. Grazer Philosophische Studien, 20(1), 265-305.

Kotzee, B. (2020). Right answer, wrong problem: commentary on A theory of moral edukation. Journal of Beliefs \& Values, 41(2), 234-236.

Larmore, Ch (1994). Strukturen moralischer Komplexität. Stuttgart: Metzler.

Luhmann, N. (1998). Die Gesellschaft der Gesellschaft. Frankfurt a. M.: Suhrkamp.

Mertens, G. (1999). Sitte und Sittlichkeit. Bedingungen ethischen Handelns in der Moderne. In V. Ladenthin \& R. Schilmöller (Hrsg.), Ethik als pädagogisches Projekt. Grundfragen schulischer Werterziehung (S. 23-42). Opladen: Leske \& Budrich.

Nassehi, A. (2017). Die letzte Stunde der Wahrheit. Kritik der komplexitätsvergessenen Vernunft (2. Aufl.). Hamburg: Murmann.

Nöstlinger, C. (1980). Moralisch unterwegs. Kursbuch, 69, 1-6.

Oelkers, J. (1992). Pädagogische Ethik. Eine Einführung in Probleme, Paradoxien und Perspektiven. Weinheim: Juventa.

Rucker, Th (2019). Erziehender Unterricht, Bildung und das Problem der Rechtfertigung. Zeitschrift für Erziehungswissenschaft, 22(3), 647-663.

De Ruyter, D. (2019). Does a theory of moral education need the input of empirical research? Journal of Philosophy of Education, 53(4), 642-648.

Scanlon, T. M. (1998). What we owe to each other. Cambridge: Harvard University Press.

Schaber, P. (2010). Wertevermittlung und Autonomie. In K. Meyer (Hrsg.), Texte zur Didaktik der Philosophie (S. 139-155). Stuttgart: Reclam.

Tenorth, H.-E. (2015). Pädagogen und Juristen - Kommunikationsprozesse zwischen feindlichen Lagern. Recht der Jugend und des Bildungswesens, 62(1), 9-16.

Thimm, A. (2007). Die Bildung der Moral. Zum Verhältnis von Ethik und Pädagogik, Erziehung und Moral. Paderborn: Schöningh.

Tugendhat, E. (1993). Vorlesungen über Ehtik. Frankfurt a. M.: Suhrkamp.

White, J. (2017). Moral education and the limits of rationality: a reply to John Tillson. Theory and Research in Education, 15(3), 339-345. 